\title{
Channel Selection and Feature Enhancement for Improved Epileptic Seizure Onset Detector
}

\author{
Marwa Qaraqe*, Muhammad Ismail ${ }^{\dagger}$, Qammer Abbasi ${ }^{\dagger}$, and Erchin Serpedin* \\ * Department of Electrical and Computer Engineering \\ Texas A\&M University, College Station, TX, 77843-3128, USA \\ Email: marwa@tamu.edu and serpedin@ece.tamu.edu \\ $\dagger^{\dagger}$ Department of Electrical and Computer Engineering \\ Texas A\&M University at Qatar, Education City, Doha, Qatar, 23874 \\ Email: m.ismail@qatar.tamu.edu and qammer.abbasi@qatar.tamu.edu
}

\begin{abstract}
This paper presents a novel architecture for a patient-specific epileptic seizure onset detector using scalp electroencephalography. The proposed architecture exploits the benefits of both channel selection and feature enhancement to improve the detector performance. The novel architecture results in higher energy difference between the pre-seizure and seizure states and hence performs better in terms of detection sensitivity and false alarm rate compared to benchmark detectors available in the literature. In detail, the proposed architecture achieves a 7\% increase in sensitivity and a reduction of 9 false alarms per hour compared to the benchmark detector.

Index Terms-epilepsy, EEG, seizure onset
\end{abstract}

\section{INTRODUCTION}

Epilepsy is a brain disorder that is characterized by intermittent abnormal firing of neurons, called seizures. Neurons normally generate electrochemical impulses that act on other neurons, glands, and muscles to produce human thoughts, feelings, and actions. However, in epilepsy, the normal rhythm of neural firing is disturbed, causing the epileptic patient to experience strange sensations, emotions, and behaviors, or sometimes convulsions, muscle spasms, and loss of consciousness [1]. Epilepsy affects approximately $1 \%$ of the population in the United States. Around $80 \%$ of those patients can control their seizures with modern medicines and surgical techniques. However, nearly 25 to 30 percent of patients are diagnosed with intractable epilepsy, where they cannot control seizures even with the best available treatment [2].

The confusion, loss of consciousness, and lack of muscle control that accompany certain types of seizures can lead to serious injuries that include fractures, head injuries, and burns. These injuries account for a significant component of the risk associated with epilepsy [3]. The risk of injury associated with epilepsy can be mitigated by using a device that can reliably detect or predict the onset of seizure episodes. Because the clinical behavior of an epileptic seizure is preceded by and then accompanied by electroencephalographic alterations, electroencephalography (EEG) can be used to measure these alterations [4].
The scalp EEG is a non-invasive, multi-electrode recording of time-varying potentials generated by the neurons located on the cerebral cortex. The electrodes are distributed symmetrically around the scalp to provide a temporal and spatial summary of the brain's electrical activity. The EEG activity of clinical relevance is limited to the frequency band $0.5-50 \mathrm{~Hz}$, and that of seizure activity is further limited to the frequency band $0.5-25 \mathrm{~Hz}$ [5].

Extensive research has been dedicated to the detection of the earliest signs of electrographic changes associated with a seizure using either scalp or inter-cranial EEG. A device that has the ability to detect the electrographic onset of a seizure will enable epileptic patients to lead a more normal and secure life, and will help them to avoid injuries due to the sudden nature of the seizure. In [7], one of the earliest automated systems for the detection of epileptic activity in long-term EEG recordings is designed and implemented by applying empirically determined thresholds on time-domain features. A seizure onset detection algorithm that processes a single, manually-selected channel of an invasive-EEG recording $(\mathrm{ECoG})$ is implemented in [8] by using a maximum-likelihood classifier with Gaussian mixture model conditional densities to differentiate between a patient's normal and abnormal ECoG. In [5], a patient-specific method for the detection of epileptic seizure onset from scalp-EEG is designed using wavelet decomposition, feature extraction, and a support vector machine (SVM) classification algorithm. In [9], automatic detection of epileptic seizure event and onset is proposed using wavelet based features and certain statistical features without wavelet decomposition.

In [10], different methods are employed for EEG channel selection preceding automatic seizure detection. Channel selection based on the highest variance method has demonstrated an improved performance compared to a no channel selection scheme. EEG channel selection reduces the detector computational complexity and avoids using channels with no relevant information that may deteriorate the detector's performance. In [11], it has been shown that EEG signal 
differentiation can be used as a feature enhancement technique for better seizure onset detection. A seizure is characterized by abnormal synchronization in neuron firing, and thus sharp spiking activities in quick succession are observed. The use of EEG differentiation accentuates the spiking activity while suppressing the background, thus aiding in the detection of seizure onset.

From [7] - [9], the seizure onset detector is traditionally composed of two stages, namely, feature extraction and classification stages. The channel selection and feature enhancement stages have been investigated separately in [10] and [11], respectively. Both stages, separately, have improved the performance of the detector. In this paper, we aim to exploit the benefits of both channel selection and feature enhancement to improve the detector performance. Hence, we propose a detector architecture that is composed of five stages, namely, EEG channel selection, feature enhancement, spatial averaging, feature extraction, and classification. Experimental results have demonstrated an improved performance for the proposed architecture as compared to a benchmark system based on [11].

The rest of this paper is organized as follows. Section II presents the proposed detector architecture. Clinical data and performance measures are presented in Section III. The results are described in Section IV. Finally, conclusions and future research are drawn in Section $\mathrm{V}$.

\section{The Detector Architecture}

This section presents the proposed detector architecture, as shown in Fig. 1, based on the following stages: channel selection, feature enhancement, spatial averaging, feature extraction, and classification.

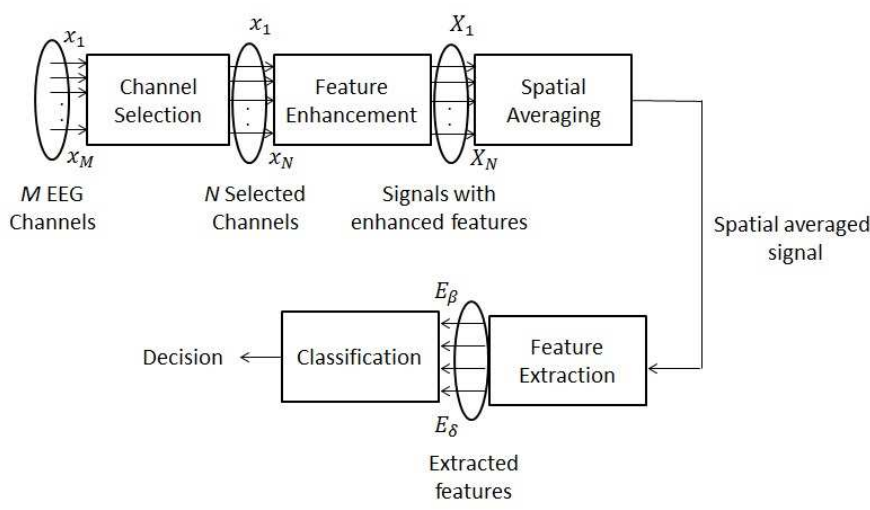

Fig. 1. The proposed detector architecture

\section{A. Channel Selection}

The task of the channel selection stage is to automatically choose the EEG channels that contain the most valuable electrographic seizure information. This in turn reduces the detector computational burden and omits invaluable channels that may deteriorate the detector's performance. Hence, given a total of $M$ EEG channels, the channel selection stage selects the $N<M$ channels with the most valuable information.

The channel selection method used in this paper is based on the variance of the EEG signal amplitude for the duration of the seizure [10]. The rationale is that during seizure a higher signal energy is expected as compared to the normal case, which can be measured through variance. The variance of channel $c, V(c)$, is expressed by,

$$
V(c)=\frac{1}{k} \sum_{i=1}^{k}\left(x_{c}(i)-\mu_{c}\right)^{2},
$$

where $x_{c}$ is the EEG data for channel $c, \mu_{c}$ is the mean of the EEG data for channel $c$, and $k$ is the number of samples in the EEG data (equal to the sampling frequency multiplied by the duration, in seconds, of the EEG data). Let $\mathcal{M}$ and $\mathcal{N}$ denote the set of available and selected EEG channels, respectively. The $\mathcal{N}$ selected channels are chosen as the ones with the highest variance values, and are given by

$$
\mathcal{N}=\arg \max V(c),
$$

where $c \in \mathcal{M}$.

\section{B. Feature Enhancement}

The role of the feature enhancement stage is to emphasize the seizure zone in the EEG data with respect to the background, which is suppressed simultaneously. In this regard, a differentiation and exponentiation approach is adopted [11], which is expressed as

$$
X_{c}(t)=\exp \left(\left(\frac{1}{w}\right)\left|\frac{d x_{c}(t)}{d t}\right|\right),
$$

where $w$ is a normalization constant ( $w=100,000$ in [11]), and $X_{c}(t)$ is the EEG signal with enhanced feature for channel $c$. The reasoning behind how (3) enhances the seizure onset with respect to the background is simple. Assuming $t_{1}, t_{2}$, and $t_{3}$ are successive time points and a spike occurs at $t_{2}$, then statistically $x_{c}\left(t_{2}\right)-x_{c}\left(t_{1}\right)$ and $x_{c}\left(t_{3}\right)-x_{c}\left(t_{2}\right)$ will have high numerical values. In the event that all three points belong to the normal, background signal, then $x_{c}\left(t_{2}\right)-x_{c}\left(t_{1}\right)$ and $x_{c}\left(t_{3}\right)-x_{c}\left(t_{2}\right)$ will have small values. As a result, $\frac{d x_{c}(t)}{d t}$ will enhance the spikes in $x$ while suppressing its background [11].

\section{Spatial Averaging}

Following the channel selection and feature enhancement stages, the next stage in the proposed detector is the spatial averaging (SA). In the SA stage, the $N$ selected channels with enhanced features are averaged so that a single EEG vector, $Y$, is obtained. SA reduces the number of features fed into the classification stage and thus reduces the detector's computational burden. For instance, if $H$ is the number of features extracted from each EEG channel, the detector must 
process $N \times H$ features. However, through SA, the detector now processes only $H$ instead of $N \times H$ features.

\section{Feature Extraction}

The onset of a seizure is often associated with rhythmic activity that is composed of multiple frequency components. As a result, different features are extracted from several EEG sub-bands for improved detection accuracy. Furthermore, since EEG is a highly non-stationary signal, features should be extracted from reasonably small time epochs. As a result, a sliding window of length $L=2$ seconds (i.e., a 2 second epoch) is passed along the EEG signal, and each epoch is fed into the feature extraction unit.

In order to extract relevant sub-band signals from the EEG epoch, a multi-resolution wavelet decomposition is used, where the low and high pass filters are chosen to be associated with the fourth member of the Daubechies wavelet family, which exhibit a maximally flat response in their pass-bands and minimum spectral leakage in their stop-bands [12]. Only the sub-band signals that collectively represent the activity at time-scales corresponding to the frequencies from 0.5 to 25 $\mathrm{Hz}$ are computed since this frequency range captures various seizure onset electrographic manifestations [13]. These subbands correspond to the $\delta, \theta, \alpha$, and $\beta$ EEG frequency bands, where $\delta<4 \mathrm{~Hz}, \theta \in[4,7] \mathrm{Hz}, \alpha \in[8,15] \mathrm{Hz}$, and $\beta \in[16,31]$ $\mathrm{Hz}$.

The extracted sub-band epoch signals are not used directly as the feature vector since the direct representation of the EEG waveform is too sensitive to noise. Instead, the energy in the two second epoch sub-bands are computed, resulting in the extraction of four energy features from a single EEG epoch, which is given by

$$
\left[\begin{array}{c}
E_{\beta} \\
E_{\alpha} \\
E_{\theta} \\
E_{\delta}
\end{array}\right]=\left[\begin{array}{l}
\sum\left(Y_{\beta}^{2}\right) \\
\sum\left(Y_{\alpha}^{2}\right) \\
\sum\left(Y_{\theta}^{2}\right) \\
\sum\left(Y_{\delta}^{2}\right)
\end{array}\right],
$$

where the summation is over the sample points in the epoch and $Y_{\beta}, Y_{\alpha}, Y_{\theta}$, and $Y_{\delta}$ are the spatially averaged signals from the $\beta, \alpha, \theta$, and $\delta$ bands, respectively.

\section{E. Classification and Detection}

In the classification stage of the detector, each EEG epoch is assigned to a seizure or non-seizure class using an SVM that is trained on feature vectors representing both seizure and non-seizure epochs. Generally, seizure and non-seizure classes are not linearly separable, thus SVM must use nonlinear decision boundaries. In the proposed detector, SVM uses a Gaussian radial basis function (RBF) kernel and a fixed set of hyperparameters (defaults in the MatLab SVM software).

The output of the SVM will declare the current epoch as seizure or non-seizure. However, in an attempt to decrease the number of false seizure detections, a timing constraint, $T$, is adopted where the detector does not declare a seizure event until SVM has detected $T=3$ consecutive seizure epochs [5].

\section{DATA AND EXPERIMENTATION}

\section{A. Clinical Data}

The data used to evaluate the proposed detector is from a publicly available database consisting of EEG recordings from pediatric subjects with intractable seizures, collected at the Children's Hospital Boston [14]. The subjects have been monitored for up to several days following withdrawal of antiseizure medication in order to characterize their seizures and assess their candidacy for surgical intervention. All signals are sampled at 256 samples per second with 16 -bit resolution. The International $10-20$ system of EEG electrode positions and nomenclature is used for these recordings. All recordings used have 23 EEG channels.

For each clinical seizure, an expert has indicated the earliest EEG change associated with the seizure. The data is segmented into one hour long records. Records that do not contain a seizure are referred to as non-seizure records and those that contain one or more seizures are referred to as seizure records.

\section{B. Testing}

To test the proposed detector, a leave-one-out crossvalidation testing scheme is adopted for each subject. In the leave-one-out cross-validation testing scheme, the SVM is given a training set that includes the seizure and non-seizure epochs from all but one of the subject's recordings. The detector then attempts to detect the seizure epochs from the excluded record using the learned knowledge from the training set. This is repeated until each recording from the subject is excluded once.

The performance of the detector is analyzed using the following measures:

- Detection latency: It is the delay between the electrographic seizure onset marked by the electroencephalographer and algorithmic seizure event declared by the detector.

- Number of false alarms (FA) per hour: It is the number of false positives per hour

- Sensitivity: It is the percentage of seizure epochs that are detected correctly

It is important to note that the performance of the detector is evaluated by looking at the classification of all epochs. No refractory period is adopted [10]. The proposed detector performance is compared to a benchmark detector based on [11], which involves a feature enhancement stage but no channel selection or spatial averaging stages.

\section{RESULTS AND DISCUSSION}

Tables I - III show the average energy difference in $\mathrm{dB}$ between the pre-seizure and seizure states for seizure records $03,04,15,16,8$ and 21 of patient CHB 01. The energy 
differences are calculated on the four extracted sub-bands. Table I shows the results when the detector only uses SA. Using only SA is somehow similar to the traditional detectors in the literature (i.e., no channel selection or feature enhancement). Table II shows the results when the detector implements the feature enhancement stage without any form of channel selection. From Tables I and II, the energy difference when feature enhancement is used is higher than when no feature enhancement is used. The energy difference is further enhanced for the case when the detector implements both feature enhancement and channel selection, as shown in Table III. It is advantageous for the energy difference between the seizure and pre-seizure states to be large since it leads to an improved detection performance, based on SVM. The ability of a SVM to discriminate between two classes is influenced by their separability. Therefore, as the difference between the seizure class and non-seizure class increases, the classifier will distinguish between the two classes more effectively, and thus the detector performs better.

The performance of the proposed detector as compared to a benchmark based on [11] is shown in Table IV. The benchmark assumes the same structure as the proposed detector but does not perform any type of channel selection. In Table IV, the results of the proposed detector are shown for 6 channels (the best detection performance is obtained by selecting 4 6 channels [10]). Comparing the two detection architectures, the proposed architecture performs better in terms of the number of false alarms detected within an hour and in terms of sensitivity. Both detectors have a very close performance in terms of detection latency, with an increase of 0.4 seconds in latency for the proposed detector.

TABLE I

Average Energy Difference between Pre-Seizure And SeIzure USING ONLY SA

\begin{tabular}{ccccccc}
\hline & Rec. 03 & Rec. 04 & Rec. 15 & Rec. 16 & Rec. 18 & Rec. 21 \\
\hline$\beta$ & 3.84 & 3.05 & 3.59 & 1.77 & 3.52 & 5.51 \\
$\alpha$ & 11.61 & 8.04 & 5.49 & 3.25 & 7.46 & 9.19 \\
$\theta$ & 12.30 & 12.15 & 7.51 & 5.27 & 9.24 & 12.08 \\
$\delta$ & 11.24 & 9.32 & 2.69 & 4.62 & 9.46 & 8.99 \\
\hline
\end{tabular}

TABLE II

Aver age Energy Difference Between Pre-SEIZure ANd SEIZure Using Feature Enhancement without Channel SELECTION

\begin{tabular}{ccccccc}
\hline & Rec. 03 & Rec. 04 & Rec. 15 & Rec. 16 & Rec. 18 & Rec. 21 \\
\hline$\beta$ & 10.66 & 11.47 & 3.14 & 9.90 & 5.18 & 6.25 \\
$\alpha$ & 13.69 & 13.22 & 4.89 & 12.44 & 10.84 & 12.16 \\
$\theta$ & 13.83 & 13.49 & 13.49 & 13.25 & 11.15 & 12.59 \\
$\delta$ & 13.42 & 15.17 & 5.83 & 15.02 & 12.19 & 12.24 \\
\hline
\end{tabular}

\section{Final Remarks AND Further Discussions}

This paper proposes a novel architecture for an epileptic seizure onset detector. The combination of the channel selection and feature enhancement stages has led to an improved
TABLE III

Average Energy Difference between Pre-Seizure and Seizure Using Feature Enhancement and Channel Selection

\begin{tabular}{ccccccc}
\hline & Rec. 03 & Rec. 04 & Rec. 15 & Rec. 16 & Rec. 18 & Rec. 21 \\
\hline$\beta$ & 12.54 & 16.44 & 5.33 & 12.56 & 11.45 & 10.94 \\
$\alpha$ & 13.61 & 18.97 & 7.13 & 13.79 & 12.39 & 12.38 \\
$\theta$ & 14.51 & 18.43 & 8.05 & 15.29 & 13.05 & 13.33 \\
$\delta$ & 14.89 & 21.66 & 7.92 & 17.58 & 14.03 & 12.61 \\
\hline
\end{tabular}

TABLE IV

Performance of the Proposed Detector as Compared to a BENCHMARK BASED ON [11]

\begin{tabular}{ccc}
\hline & No Ch. Selection & With Channel Selection \\
\hline Detection Latency & $3.6 \mathrm{sec}$ & $4 \mathrm{sec}$ \\
FAs per hour & 137.8 & 128 \\
Sensitivity & $80.6 \%$ & $87.5 \%$ \\
\hline
\end{tabular}

detection performance. An increase in the energy difference between seizure and pre-seizure states is observed when the proposed detection is implemented, versus implementing the detection system without any form of channel selection. The proposed system also performed better in terms of false alarm rate and sensitivity.

\section{ACKNOWLEDGMENT}

This publication was made possible by NPRP grant NPRP 6 - 415 - 3 - 111 from the Qatar National Research Fund (a member of Qatar Foundation). The statements made herein are solely the responsibility of the authors.

\section{REFERENCES}

[1] L. D. Iasemidis, "Epileptic seizure prediction and control," IEEE Transactions on Biomedical Engineering, vol. 50, no. 5, pp. 549-558, 2003.

[2] I. E. Leppik, Contemporary diagnosis and management of the patient with epilepsy, Handbooks in Health Care Newton, PA, 1998.

[3] T. A. Betts, "Epilepsy at the millennium," Seizure, vol. 8, no. 5, pp. 259260, 1999.

[4] B. Abou-Khalul and K. E. Misulis, Atlas of EEG \& Seizure Semiology: Text with DVD, Butterworth-Heinemann, 2005.

[5] A. Shoeb, H. Edwards, J. Connolly, B. Bourgeois, S. T. Treves, and J. Guttag, "Patient-specific seizure onset detection," Epilepsy \& Behavior, vol. 5, no. 4, pp. 483-498, 2004.

[6] H. Qu and J. Gotman, "A patient-specific algorithm for the detection of seizure onset in long-term EEG monitoring: possible use as a warning device," IEEE Transactions on Biomedical Engineering, vol. 44, no. 2, pp. 115-122, 1999.

[7] J. Gotman and P. Gloor, "Automatic recognition and quantification of interictal epileptic activity in the human scalp EEG," Electroencephalography and clinical Neurophysiology, vol. 41, no. 5, pp. 513-529, 1976.

[8] L. Meng, M. G. Frei, I. Osorio, G. Strang, and T. Q. Nguyen, "Gaussian mixture models of ECoG signal features for improved detection of epileptic seizures," Medical engineering \& physics, vol. 26, no. 5, pp. 379-393, 2004

[9] N. Ahammad, T. Fathima, and P. Joseph, "Detection of Epileptic Seizure Event and Onset Using EEG," BioMed research international, vol. 2014, 2014.

[10] J. Duun-Henriksen, T. W. Kjaer, R. E. Madsen, L. S. Remvig, C. E. Thomsen, and H. B. D. Sorensen, "Channel selection for automatic seizure detection," Clinical Neurophysiology, vol. 123, no. 1, pp. 84-92, 2012 . 
[11] K. K. Majumdar and P. Vardhan, "Automatic seizure detection in ECoG by differential operator and windowed variance," IEEE Transactions on Neural Systems and Rehabilitation Engineering, vol. 19, no. 4, pp. 356$365,2011$.

[12] G. Strang and T. Nguyen, Wavelets and filter banks, SIAM, 1996.

[13] J. Gotman, "Automatic recognition of epileptic seizures in the EEG," Electroencephalography and clinical Neurophysiology, vol. 54, no. 5, pp. 530-540, 1982.

[14] A. L. Goldberger, L. AN. Amaral, L. Glass, J. M. Hausdorff, P. Ch. Ivanov, R.G. Mark, J. E. Mietus, G. B. Moody, C. K. Peng, and H. E. Stanley, "Physiobank, physiotoolkit, and physionet components of a new research resource for complex physiologic signals," Circulation, vol. 101, no. 23 , pp. 215-220, 2000. 